\title{
A homozygous mutation in the insulin gene (INS) causing autosomal recessive neonatal diabetes in Saudi families
}

\author{
Adnan Al Shaikh, MD', \\ Bader Shirah, MS', \\ Somaya Alzelaye, $\mathrm{MD}^{2}$
}

'King Abdulaziz Medical City, King Abdullah International Medical Research Center, King Saud bin Abdulaziz University for Health Sciences, Jeddah, Saudi Arabia

${ }^{2}$ Pediatric Endocrine \& Diabetes Consultant, Endocrine \& Diabetes Center, Alqounfudah General Hospital, Al Qunfudah, Saudi Arabia

\begin{abstract}
Purpose: Insulin gene (INS) mutations are a rare cause of permanent neonatal diabetes and mature-onset diabetes of the young (MODY10). Homozygous mutations have been reported to cause diabetes by decreasing insulin biosynthesis through distinct mechanisms. In this study, we report a homozygous mutation c.$331 \mathrm{C}>\mathrm{G}$ in the INS gene causing autosomal recessive neonatal diabetes in Saudi families and share our experience with diagnosis and management.

Methods: We retrospectively reviewed all cases diagnosed with diabetes during the first week of life. We identified 18 cases, and all underwent genetic testing to identify the cause. Most had mutations in common genes (9 in KCNJ11 and 5 in $A B C(8)$. The inclusion criterion for this study was a mutation in the INS gene.

Results: Four patients from 3 Saudi families had mutations in the INS gene. All patients were born with low birth weight and were diagnosed with neonatal diabetes at the age of 2 days. Sanger sequencing analysis identified a homozygous INS pathogenic promoter variant, c.-331C $>$ G. All patients were managed by insulin therapy. Two patients had persistent diabetes and in 2 cases diabetes resolved.

Conclusion: This report indicates that a homozygous mutation in the INS gene is a probable and important cause of neonatal diabetes in Saudi Arabia. The c.-331C>G variant in the INS gene identified in our study showed variability both within and between families and different outcomes ranging from early resolution of diabetes after 2 months of life to permanent diabetes.
\end{abstract}

Keywords: INS, Autosomal recessive, Neonatal diabetes, Saudi Arabia

\section{Introduction}

Neonatal diabetes mellitus is defined as hyperglycemia lasting for more than 2 weeks and occurring within the first 6 months of life. ${ }^{1)}$ Two main clinical types of neonatal diabetes exist; permanent neonatal diabetes and transient neonatal diabetes. ${ }^{2)}$ Insulin gene (INS) mutation is a rare cause of permanent neonatal diabetes and mature-onset diabetes of the young (MODY10). ${ }^{3)}$ Heterozygous missense mutations in the insulin gene have been reported to cause permanent neonatal diabetes mellitus by preventing normal folding of proinsulin, which leads to beta-cell death through endoplasmic reticulum stress and apoptosis. ${ }^{4}$ Homozygous mutations can also cause diabetes by decreasing insulin biosynthesis through distinct mechanisms, including gene deletion, lack of translation initiation signaling, and altered mRNA stability because of the disruption of a polyadenylation signal. ${ }^{5)}$

Mutations in the INS gene were first reported to cause neonatal diabetes by Støy et al. ${ }^{2)}$ in 2007, who reported 10 new mutations in 16 probands with neonatal diabetes. Since then, several studies have reported more than 50 mutations in the INS gene with both homozygous and heterozygous modes of inheritance that cause a variety of phenotypes including https://orcid.org/0000-0002-24329308 
permanent neonatal diabetes, transient neonatal diabetes, and MODY 10. ${ }^{6}$ In this article, we aim to report 3 Saudi families with homozygous mutations in the INS gene causing neonatal diabetes and share our experience in the diagnosis and management of these rare cases.

\section{Materials and methods}

We retrospectively reviewed all cases who were diagnosed with diabetes during the first week of life at King Abdulaziz Medical City in Jeddah and Alqunfudah General Hospital in Al Qunfudah, Saudi Arabia between 2004-2019. We identified 18 cases, all of which underwent genetic testing to identify the cause. Most had mutations in common genes (9 in KCNJ11 and 5 in $A B C C 8$ ), and only 4 patients from 3 Saudi families had mutations in the INS gene. The inclusion criterion for this study was a mutation in the INS gene. Patients who had neonatal diabetes due to other causes were excluded. The pedigrees of the 3 families are shown in Fig. 1. The patients were fully evaluated with serial blood tests and genetic analysis. Blood samples (3-5 $\mathrm{mL}$ of blood) were collected in ethylene diamine tetraacetic acid (EDTA) tubes and sent fresh (not frozen) at room temperature. Genomic DNA was obtained from the blood. The samples were tested for the most common causes of neonatal diabetes (mutations in the ABCC8, KCNJ11, and INS genes) by Sanger sequencing. If a causative mutation was not identified after this first test, the patient's sample was tested for mutations in all known neonatal diabetes genes using a targeted next-generation sequencing assay. The patients were followed by a pediatric endocrinologist and were managed with different insulin therapy protocols. The variables collected in the present study included age, sex, and clinical data, investigations, treatments, and outcomes. This study was approved by the Institutional Review Board (IRB) of King Abdullah International Medical Research Center under IRB number IRBC/0657/19. The families consented to genetic testing after explanation of the pros and cons of such investigations. Genetic testing was performed at the University of Exeter Medical School, UK which was funded by the Wellcome Trust.

\section{Results}

All patients were born with low birth weight (mean, $1.69 \pm 0.14$ $\mathrm{kg}$ ) and were diagnosed with neonatal diabetes at the age of 2 days based on elevated blood glucose levels (more than $200 \mathrm{mg}$ / dL). All patients were born to consanguineous parents that had a positive family history of diabetes. Gestational diabetes was
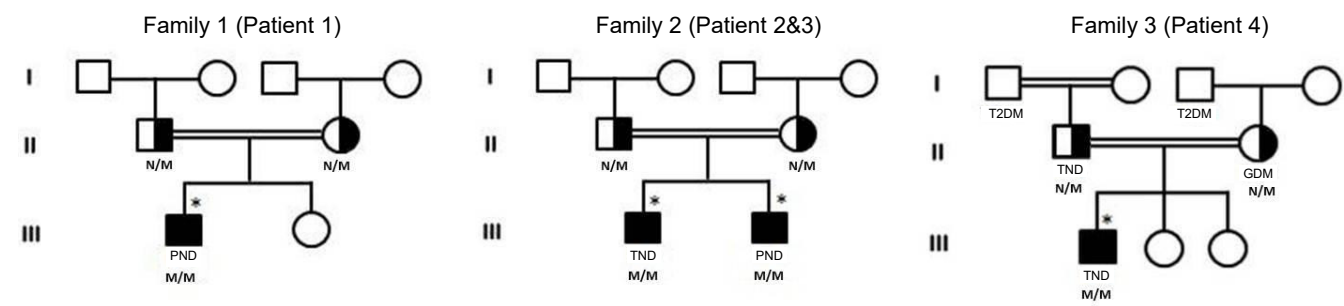

Fig. 1. Family pedigree. The samples marked with asterisks are affected individuals. TND, transient neonatal diabetes; PND, permanent neonatal diabetes; GDM, gestational diabetes mellitus; T2DM, type 2 diabetes mellitus; N, normal allele; M, mutation.

Table 1. Clinical Information of the study population

\begin{tabular}{|c|c|c|c|c|}
\hline Variable & Patient 1 & Patient 2 & Patient 3 & Patient 4 \\
\hline Age at diagnosis (day) & 2 & 2 & 2 & 2 \\
\hline Birth weight (kg) & 1.80 & 1.27 & 1.78 & 1.89 \\
\hline C-peptide level (pmol/L) & $<33$ & $<33$ & $<166$ & Not done \\
\hline Insulin level (IU/mL) & 1.7 & 1.62 & 7.4 & 1.23 \\
\hline Islet cell antibodies & Not done & Not done & Negative & Not done \\
\hline Celiac screening & Negative & Positive & Positive & Not done \\
\hline $\begin{array}{l}\text { Blood glucose at the time of diagnosis } \\
(\mathrm{mg} / \mathrm{dL})\end{array}$ & 265 & 302.4 & 219.6 & 232 \\
\hline $\mathrm{HbA1c}$ at the time of diagnosis (\%) & 5.80 & 5.70 & 6.10 & 5 \\
\hline Family history of diabetes & Positive & Positive & Positive & Positive \\
\hline Mother with gestational diabetes & No & No & No & Yes \\
\hline Parents consanguinity & Yes & Yes & Yes & Yes \\
\hline Treatment & Insulin (glargine + regular) & Insulin (glargine + regular) & Insulin (glargine + regular) & Insulin (NPH + aspart) \\
\hline Outcome & Persistent & Persistent & $\begin{array}{l}\text { Resolved at the age of } \\
10 \text { months }\end{array}$ & $\begin{array}{l}\text { Resolved at the age of } \\
2 \text { months }\end{array}$ \\
\hline Current age & $15 \mathrm{yr}$ & $9 \mathrm{yr}$ & $14 \mathrm{yr}$ & $8 \mathrm{mo}$ \\
\hline
\end{tabular}

HbA1c, glycosylated hemoglobin; NPH, neutral protamine hagedorn . 
diagnosed in only one of the mothers (patient 4). C-peptide level was reduced in 3 patients and not measured in 1 patient. Insulin level was reduced in 2 patients and not measured in 2 patients. All patients were managed by insulin therapy (mainly glargine+regular). Two patients had persistent diabetes and diabetes resolved in 2 patients (at the ages of 2 months and 10 months, respectively). The detailed clinical information of the participants is summarized in Table 1.

Sanger sequencing analysis of coding and flanking intronic regions of KCNJ11 (NM_000525.3), INS (NM_001185098.1), and ABCC8 (NM_001287174.1) genes identified a homozygous INS pathogenic promoter variant, c.-331C $>\mathrm{G}$ (Fig. 2). This variant disrupts a transcriptional regulatory site and is likely to result in decreased insulin transcription. ${ }^{5}$ The variant identified in our patients was classified as pathogenic considering the current evidence of the variant (established association between the gene and patient phenotype, rarity in control populations, functional assay data, identification of the variant in homozygous state in previous families, respectively). This result confirmed the diagnosis of recessively inherited neonatal diabetes due to pathogenic variants in the INS gene. Segregation studies were performed on the parents and identified a heterozygous carrier state in all parents.

\section{Discussion}

Homozygous mutations represent around 20\% of mutations in the INS gene and are considered "loss-of-function" mutations that affect insulin biosynthesis at transcriptional and/ or translational levels by a variety of mechanisms. ${ }^{7)}$ These mutations can result in a more than $80 \%$ reduction in insulin production in heterozygous individuals. The remaining normal INS gene allele is adequate to maintain normoglycemia in affected individuals. ${ }^{8)}$ The typical phenotype of homozygous mutations is permanent neonatal diabetes, but it also can manifest as transient neonatal diabetes or diabetes outside the neonatal period. ${ }^{9}$

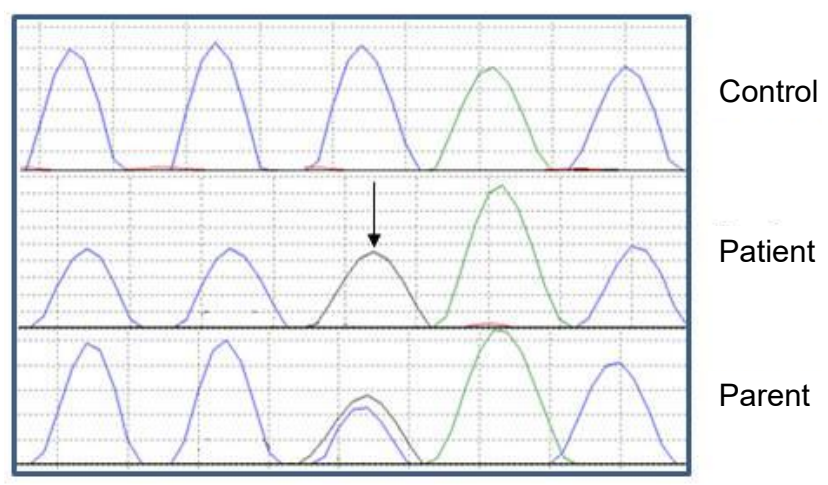

Fig. 2. The arrow indicates the homozygous C.-331C $>$ G INS mutation identified by Sanger sequencing in the proband. Sequence traces for a parent who is a heterozygous carrier for the same mutation and an unaffected control are also provided.
The typical clinical manifestations of recessive INS mutations include marked reduction in birth weight, early-onset diabetes (median 1 week), ketoacidosis, insulin deficiency, and absence of extrapancreatic features. Neonates usually have a low birth weight, which is due to insulin deficiency affecting growth and development. ${ }^{10)}$ Earlier diagnosis of diabetes and lower birth weight in patients with recessive INS mutations is due to differences in the underlying pathophysiology. Recessive mutations lead to disrupted insulin synthesis as soon as fetal beta cells start to secrete insulin. On the other hand, patients with heterozygous mutations require insulin secretion before beta-cell dysfunction develops. This eventually leads to destruction of beta cells through stress resulting from accumulation of the misfolded preproinsulin peptide in the endoplasmic reticulum. ${ }^{11)}$ The clinical manifestations of our patients (low birth weight, early age of diagnosis, and variable prognosis as transient or permanent) were similar to previously published cases with recessive INS mutations.

Clinical presentations and outcomes vary among individuals with recessive INS gene mutations, ranging from severe neonatal onset to a mild adult-onset form of the disease. Outcomes also vary, with some patients having lifelong disease and others being able to sustain normoglycemia without the use of exogenous insulin after a period of time. ${ }^{12)}$ This indicates that mutations in the INS gene have variable expression, which is usually influenced by the nature and location of the mutation. However, variability both within and between families with the same mutation has also been observed. ${ }^{13)}$ Our experience supports these findings, since the same mutation occurred in all individuals but outcomes ranged from early resolution of diabetes after 2 months of life to permanent diabetes.

Saudi Arabia has a high rate of consanguinity that ranges from $25 \%$ to $65 \%$. ${ }^{14)}$ Consanguinity is well-known to favor the occurrence of autosomal recessive diseases. Appropriate education with a special emphasis on this is needed in Saudi communities. Genetic screening in the premarital or preimplantation periods enables early genetic diagnosis and is of paramount importance in helping couples who carry the same disease-causing variants make informed decisions. ${ }^{15}$ )

In conclusion, this study indicates that a homozygous mutation in the INS gene is a probable and important cause of neonatal diabetes in Saudi Arabia. The c. $-331 \mathrm{C}>\mathrm{G}$ variant in the INS gene identified in our study showed variability both within and between families and different outcomes ranging from early resolution of diabetes after 2 months of life to permanent diabetes. To the best of our knowledge, this is the first study from Saudi Arabia to report this rare mutation and to highlight the importance of genetic testing in identifying the cause of neonatal diabetes. Further studies are needed to expand our understanding of the genetic spectrum of neonatal diabetes in Saudi Arabia. 


\section{Conflict of interest}

No potential conflict of interest relevant to this article was reported.

\section{References}

1. Meur G, Simon A, Harun N, Virally M, Dechaume A, Bonnefond A, et al. Insulin gene mutations resulting in early-onset diabetes: marked differences in clinical presentation, metabolic status, and pathogenic effect through endoplasmic reticulum retention. Diabetes 2010;59:653-61.

2. Støy J, Edghill EL, Flanagan SE, Ye H, Paz VP, Pluzhnikov A, et al. Insulin gene mutations as a cause of permanent neonatal diabetes. Proc Natl Acad Sci U S A 2007;104:15040-4.

3. Boesgaard TW, Pruhova S, Andersson EA, Cinek O, Obermannova B, Lauenborg J, et al. Further evidence that mutations in INS can be a rare cause of MaturityOnset Diabetes of the Young (MODY). BMC Med Genet 2010;11:42.

4. Polak M, Dechaume A, Cavé H, Nimri R, Crosnier H, Sulmont V, et al. Heterozygous missense mutations in the insulin gene are linked to permanent diabetes appearing in the neonatal period or in early infancy: a report from the French ND (Neonatal Diabetes) Study Group. Diabetes 2008;57:1115-9.

5. Garin I, Edghill EL, Akerman I, Rubio-Cabezas O, Rica I, Locke JM, et al. Recessive mutations in the INS gene result in neonatal diabetes through reduced insulin biosynthesis. Proc Natl Acad Sci U S A 2010;107:3105-10.

6. Piccini B, Artuso R, Lenzi L, Guasti M, Braccesi G, Barni F, et al. Clinical and molecular characterization of a novel INS mutation identified in patients with MODY phenotype. Eur J Med Genet 2016;59:590-5.
7. Liu M, Sun J, Cui J, Chen W, Guo H, Barbetti F, et al. INSgene mutations: from genetics and beta cell biology to clinical disease. Mol Aspects Med 2015;42:3-18.

8. Liu M, Hodish I, Haataja L, Lara-Lemus R, Rajpal G, Wright J, et al. Proinsulin misfolding and diabetes: mutant INS gene-induced diabetes of youth. Trends Endocrinol Metab 2010;21:652-9.

9. Ivarsson SA, Lernmark A. Comment on: Edghill et al. (2008) Insulin mutation screening in 1,044 patients with diabetes: mutations in the INS gene are a common cause of neonatal diabetes but a rare cause of diabetes diagnosed in childhood or adulthood: Diabetes 57:1034-1042, 2008. Diabetes 2008;57:e9.

10. Raile K, O'Connell M, Galler A, Werther G, Kühnen P, Krude H, et al. Diabetes caused by insulin gene (INS) deletion: clinical characteristics of homozygous and heterozygous individuals. Eur J Endocrinol 2011;165:25560.

11. Støy J, Steiner DF, Park SY, Ye H, Philipson LH, Bell GI. Clinical and molecular genetics of neonatal diabetes due to mutations in the insulin gene. Rev Endocr Metab Disord 2010;11:205-15.

12. Courtney R, Gamble C, Arango ML, Shah A, Rubio NI, Nguyen J, et al. Novel homozygous likely-pathogenic intronic variant in INS causing permanent neonatal diabetes in siblings. J Pediatr Endocrinol Metab 2016;29:1089-93.

13. Nishi M, Nanjo K. Insulin gene mutations and diabetes. J Diabetes Investig 2011;2:92-100.

14. Warsy AS, Al-Jaser MH, Albdass A, Al-Daihan S, Alanazi M. Is consanguinity prevalence decreasing in Saudis? A study in two generations. Afr Health Sci 2014;14:314-21.

15. Alharbi OA, Al-Shaia WA, Al-Hamam AA, Al-Marzoug HM, Ahmed AE, Bagha M. Attitude of Saudi Arabian adults towards consanguineous marriage. Qatar Med J 2015;2015:12. 